\title{
Epidemiology and Sensibility Profile of Some Enteric Bacteria Isolated from Stool Samples in Some Douala-Cameroon Hospitals
}

\author{
Raphael Tchientcheu ${ }^{1}$, Calixte Ida Penda ${ }^{2,3}$, Rebecca Madeleine Ebelle Etame ${ }^{1}$, \\ Colette Grace Ngondi Dalle ${ }^{3}$, Raymond Simplice Mouokeu ${ }^{4, *}$, Rosalie Anne Ngono Ngane ${ }^{1}$ \\ ${ }^{1}$ Department of Biochemistry, Faculty of Science, University of Douala, Douala, Cameroon \\ ${ }^{2}$ Department of Clinical Sciences, Faculty of Medicine and Pharmaceutical Sciences, University of Douala, Douala, Cameroon \\ ${ }^{3}$ Department of Pediatry, Laquintinie Hospital, Douala, Cameroon \\ ${ }^{4}$ Institute of Fisheries and Aquatic Sciences, University of Douala, Douala, Cameroon
}

\section{Email address:}

moraysi@yahoo.fr (R. S. Mouokeu)

${ }^{*}$ Corresponding author

\section{To cite this article:}

Raphael Tchientcheu, Calixte Ida Penda, Rebecca Madeleine Ebelle Etame, Colette Grace Ngondi Dalle, Raymond Simplice Mouokeu, Rosalie Anne Ngono Ngane. Epidemiology and Sensibility Profile of Some Enteric Bacteria Isolated from Stool Samples in Some DoualaCameroon Hospitals. International Journal of Gastroenterology. Vol. 5, No. 1, 2021, pp. 5-17. doi: 10.11648/j.ijg.20210501.12

Received: December 21, 2020; Accepted: December 29, 2020; Published: January 22, 2021

\begin{abstract}
Background: Gastroenteritis caused by bacteria are a serious public health issue. Antibiotic resistance is common. This work described the epidemiological and resistance profile of bacteria involved in gastrointestinal infection in Douala, Cameroon. Methods: A cross-sectional study was conducted from March to December 2017 on stool samples. Bacterial species were diagnosed on the basis of morphological, biochemical and physiological tests. The resistance profile on fifteen routine antibiotics was investigated using the disks diffusion method. Results: Out of 250 stool samples examined, 206 were positive, 129 $(62.62 \%)$ samples had only one bacterial species and 77 (37.38\%) more than one species. 301 pathogenic bacteria were identified of which Escherichia coli represented more than one third (33.89\%), followed by Klebsiella pneumoniae (22.26\%), Enterobacter aerogenes (11.96\%), Salmonella sp (10.30\%), Citrobacter freundii (9.97\%), Yersinia enterocolitica (4.65\%), Shigella flexneri (3.99\%), Serratia marcescens (2.33\%) and Proteus sp (0.66\%). The antibiogram showed high resistance to Tetracycline, Amoxycillin, Amoxycillin + clavulanic acid, Doxycycline and Cotrimoxazol. Imipenem and Amikacin were the most active Conclusion: The present findings provide additional information for the control of gastrointestinal tract infections in Douala.
\end{abstract}

Keywords: Epidemiology, Gram- Bacteria, Gastroenteritis, Antibiotic Resistance, Douala, Cameroon

\section{Introduction}

Bacteria is one of the main causes of infection in humans [1]. More specifically, enteric diseases caused by bacteria constitute the main cause of morbidity and mortality in low income countries and represent approximately $50 \%$ of nosocomial infections. Children are considered the most vulnerable group to bacterial infections [2]. According to the World Health Organization, the resistance of the bacteria remains a major cause of morbidity and mortality worldwide [3]. Antimicrobial resistance (AMR) among human pathogens remains a major concern for health problems [4]. In the whole world, resistance to existing antibiotics is growing at an alarming rate [5]. Based on a recent study, 700.000 deaths are reported annually due to AMR around the world, and it has been predicted that if appropriate control and preventive measures are not taken, AMR would become one of the main reasons for deaths among hospitalized or non-hospitalized patients in both developing and developed countries [6]. Proper use of antibiotics is essential for treating bacterial infections [7]. Thus, inappropriate prescriptions and misuse of antibiotics contribute to the emergence of AMR pathogenic bacteria, restriction of therapeutic options, increase in hospitalization period, high treatment costs and finally a greater death rate [8]. According to the global action plan on AMR endorsed by the World Health 
Organization (WHO), it is important to raise awareness of AMR by monitoring research programs in different parts of the world [9]. AMR monitoring is critical and has several benefits including 1) providing data on bacterial resistance rate 2) helping select appropriate antibiotics and subsequently reduce AMR [10], 3) reducing hospitalizations and treatment costs and 4) decrease in death rates [7]. In Africa and in Cameroon in particular, infectious diseases such as gastroenteritis remain endemic and represent one of the highest reasons for consultation or hospital visits. Recent studies conducted in Cameroon have shown Enterobacteriaceae producing broadspectrum beta-lactamases in the cities of Yaounde and Ngaoundere [11]. An antibiotic resistance prevalence of $7.9 \%$ and $22.6 \%$ respectively to Gentamycin and Ciprofloxacin was found among patients in consultations at the Dschang District Hospital [12]. However, data on antibiotic resistance remain limited and the epidemiology of bacteria is unknown while the species prevalence varies with time. In addition, many patients do not have access to medical laboratories and the management of gastroenteritis remain problematic [13]. Isolation and antibiogram are rarely requested, consequently, the data on the epidemiology of enteric bacteria and their antibiotic resistance are therefore patchy. Therefore, it is important to have a reliable database for this phenomenon to improve patient care. This study was conducted to diagnose common bacteria involved in enteric infections in Douala (Cameroon) and to investigate their susceptibility to routine antibiotics.

\section{Materials and Methods}

\subsection{Study Design}

A cross-sectional study was carried out from March to December 2017 on the distribution and resistance profile of enteric bacteria to commonly used antibiotics. The investigations were carried out in 3 hospitals in the city of Douala (Littoral Region, Cameroon): Laquintinie Hospital, Deido District Hospital and Bonamoussadi District Medical Center.

\subsection{Study Population}

All patients of any age complaining of abdominal pain who attended the above hospitals and received a prescription for stool examinations were included after obtaining informed consent. For each patient, a standardized interview was carried out and their medical file consulted in order to document the socio-demographic data (age, sex, place of residence).

\subsection{Ethical Considerations}

The study was carried out according to the guidelines for human experimental models in clinical research as stated by the Cameroon Ministry of Public Health. Besides, the ethical and administrative clearances for this study were issued by the institutional ethic committee of the University of DoualaCameroon (CEI-UDo) under reference number CEIUDo/691/11/2016/T of $24^{\text {th }}$ November 2016. This was followed by the administrative agreement of the Regional Delegate of the Ministry of Public Heath in Littoral Region on the $2^{\text {nd }}$ February 2017 and the research authorization of the administrative authority of the institution where samples collection was conducted. Only patients who signed an informed consent form for their participation were enrolled. Participation in the study was strictly voluntary and they were free to decline answering any question or totally withdraw if they so wished at any time.

\subsection{Bacterial Isolation and Identification}

\subsubsection{Collecting Stool Samples}

All patient's hands were washed aseptically and then rinsed with tap water. Subsequently, approximately $10 \mathrm{~g}$ of stool was obtained in a sterile bottle. The bottle was closed hermetically, labeled and transported in a cooler to the laboratory for bacterial analyses within 2 hours from collection. These samples were taken according to the recommendations of the Reference in medical microbiology (REMIC) [14]. Each sample obtained was observed under a light microscope (Olympus XSZ-107BN; x40) in a fresh state, as well as at colored state following Gram staining to detect the presence of the different bacteria types (Gram positive and/or Gram negative).

\subsubsection{Bacterial Isolation and Identification}

Different selective and differential culture media were used. These media were Salmonella Shigella agar (SS agar), Hektoen, Cetrimide, Yersinia CIN agar, Eosin Methylen Blue (EMB) agar, MacConkey agar, Nutritive agar, Nutrient broth, Selenite broth and Peptone broth (ACCUMiX ${ }^{\mathrm{TM}}$, TULIP DIAGNOSTICS (P) LTD, Verna Industrial Estate, India). All of these culture media were prepared as prescribed by the manufacturers. Those containing the agar were each poured into a three-compartment Petri dish ( $9 \mathrm{~cm}$ in diameter). Two suspensions of each stool sample were prepared in $10 \mathrm{ml}$ of both peptone broth and Selenite Broth (pre-enrichment). Each of the first suspensions was seeded on the solid media indicated above. The other preenriched suspension was sown on SS and Hektoen media [15]. The seeding was done by the dial method and the Petri dishes were incubated $24 \mathrm{~h}$ at $37^{\circ} \mathrm{C}$. At the end of the incubation time, the bacterial colonies were observed. Black, colorless, or colorless with gray center colonies on SS agar were considered to be Salmonella sp, Shigella sp or Serratia sp respectively. Green colonies with metallic reflect on EMB and which after subculture on MacConkey agar appeared in discrete pinkish colonies were considered to be Escherichia coli. The colonies of Klepsiella sp, Citrobacter sp and Enterobacter sp were respectively pink with a violet convex center without metallic surfaces, pale violet with a metallic character and bluish aspect on EMB agar. The creamy to yellow colonies on Cetrimide were considered to be Pseudomonas sp. On Hektoen, the colonies of Proteus sp were yellow with a black center. The colonies of Yersinia enterocolitica were pale pink and dark red in the center (bull's eye colony) on Yersinia CIN (Cefsulodine-IrgasanNovobiocine) agar [15]. All the isolated colonies were successively subcultured on Ordinary Nutrient Agar (ONG) until pure colonies were obtained. The pure colonies obtained were subjected to various tests of morphocultural and biochemical characterizations (Catalase, Oxidase, ONPG, 
Mannitol mobility, Urea-indol...) before being confirmed on the API gallery (20E). These isolated and identified clinical isolates were stored at $-80^{\circ} \mathrm{C}$ in Cryotubes containing Brain Heart Broth and $20 \%$ glycerol.

\subsection{In vitro Evaluation of the Susceptibility of Isolated Bacteria to Conventional Antibiotics}

The susceptibility tests of the various isolates obtained was performed by the disk diffusion method (Kirby-Bauer) on Mueller Hinton Agar (MHA) according to standard procedures prescribed by CASFM [16]. All antibiotics used in these assays were the most common antibacterial agents that are commonly used by physicians. The list was provided by physicians and nurses that work for the targeted healthcare facilities. Thus, fifteen routine antibiotics purchased at Sigma-Aldrich; France, were used. Antibacterial susceptibility was carried out according to the protocol recommended by EUCAST/SFM (European Committee on Antimicrobial Susceptibility Testing, 2015) with some modifications. Table 1 presents the different antibiotics used with their concentrations and their activity scale. From a 24 h bacterial cell culture carried out on Mueller Hinton Agar (MHA), a suspension of each bacteria was prepared in $10 \mathrm{ml}$ sterile distilled water. The turbidity of each suspension was adjusted to match the 0.5 scale of McFarland as recommended by CASFM [16] for non-stringent bacteria. The bacterial suspension was then diluted 100 times. One milliliter of each bacterial suspension was aseptically spread over the surface of a $90 \mathrm{~mm}$ Petri dish containing MHA. The Petri dishes were left to dry for $5 \mathrm{~min}$. Then, the antibiotics discs were placed on the agar surface. $30 \mathrm{~min}$ after the application of the discs, the Petri dishes were incubated $24 \mathrm{~h}$ at $37^{\circ} \mathrm{C}$. At the end of the incubation period, the diameters of the inhibition zones were measured. These diameters were interpreted according to the scale sensitivity (Sensitive-Intermediate-Resistant) in accordance with the CASFMI recommendations [16].

Table 1. Antibiotics used with their critical diameters.

\begin{tabular}{|c|c|c|c|c|c|}
\hline \multirow{2}{*}{ Antibiotics group } & \multirow{2}{*}{ Types of Antibiotics } & \multirow{2}{*}{ Charge of disc } & \multicolumn{3}{|c|}{ Critical diameters of antibiotics $(\varnothing \mathrm{mm})$} \\
\hline & & & $\mathbf{S}$ & I & $\mathbf{R}$ \\
\hline \multirow{4}{*}{$\beta$-lactamins } & Amoxicillin (AX) & $25 \mu \mathrm{g}$ & $\geq 21$ & $16<\emptyset \leq 21$ & $<16$ \\
\hline & Amoxicillin/clavulanic acid (AMX) & $20 / 10 \mu \mathrm{g}$ & $\geq 24$ & $16<\emptyset \leq 24$ & $<16$ \\
\hline & Imipenem (IMP) & $10 \mu \mathrm{g}$ & $\geq 16$ & $14<\emptyset \leq 15$ & $\leq 13$ \\
\hline & Ceftazidim (CAZ) & $5 \mu \mathrm{g}$ & $\geq 19$ & $16<\emptyset \leq 18$ & $\leq 15$ \\
\hline \multirow{2}{*}{ Aminoside } & Gentamicin $(\mathrm{CN})$ & $15 \mu \mathrm{g}$ & $\geq 18$ & $16<\emptyset \leq 18$ & $<16$ \\
\hline & Amikacin (AK) & $30 \mu \mathrm{g}$ & $\geq 17$ & $15<\emptyset \leq 17$ & $\leq 15$ \\
\hline \multirow{2}{*}{ Sulfamides } & Polymixin Colistin (CT) & $10 \mu \mathrm{g}$ & $\geq 15$ & & $<15$ \\
\hline & Cotrimoxazole (STX) & $25 \mu \mathrm{g}$ & $\geq 16$ & $13<\emptyset \leq 16$ & $<13$ \\
\hline \multirow[t]{2}{*}{ Phenicols } & Chloramphenicol (CHL) & $30 \mu \mathrm{g}$ & $\geq 23$ & $23<\emptyset \leq 23$ & $<23$ \\
\hline & Ciprofloxacin (CIP) & $5 \mu \mathrm{g}$ & $\geq 25$ & $22<\emptyset \leq 25$ & $<22$ \\
\hline \multirow[t]{2}{*}{ Fluoroquinolones } & Norfloxacin (NOR) & $10 \mu \mathrm{g}$ & $\geq 16$ & $13<\emptyset \leq 16$ & $<12$ \\
\hline & Nalidic Acid (NA) & $30 \mu \mathrm{g}$ & $\geq 20$ & $15<\emptyset \leq 20$ & $<20$ \\
\hline \multirow{2}{*}{ Cyclins } & Tetracycline (TE) & 30 UI & $\geq 19$ & $17<\emptyset \leq 19$ & $<17$ \\
\hline & Doxycycline (DO) & $30 \mathrm{UI}$ & $\geq 19$ & $17<\emptyset \leq 19$ & $<17$ \\
\hline
\end{tabular}

Sensible (S), Intermediate (I) and Resistant (R)

\subsection{Statistical Analyses}

All data were verified for consistency, coded, and keyed in an Excel sheet. Data were summarized in table as percentages with $95 \%$ confidence interval $(95 \% \mathrm{CI})$ or mean \pm standard deviation (SD) for qualitative and quantitative variables respectively where appropriate.

\section{Results}

\subsection{Socio-demographic Characteristics of the Studied Population}

According to the percentage of samples received per center, Laquintinie and Bonamoussadi hospitals received more patients for stools examination than others (100 and 86 respectively). The percentage of male and women who contract digestive tract infections was 50.5\% (104/206) and $49.5 \%$ (102/206) respectively, sex-ratio was therefore 1.02 .

Figure 1a shows the distribution of individuals relevant to age group. This figure revealed that children among 0 to 5 years were the most infected (31.06\%), followed by adults among 25 to 40 years $(25.26 \%)$ and the patients least recorded were above 65 years (3.75\%). Similarly, the distribution of patients according to gender and age group shows that men are the most represented in the age group between 0 and 25 years while up to 25 years, women are more effective within all classes (Figure 1b).

\subsection{Frequency of Identification of Bacteria Species}

About 301 bacteria were isolated among which 152 and 149 from men and women respectively. These isolates were characterized and identified as belonging to the genus Citrobacter, Enterobacter, Escherichia, Klepsiella, Proteus, Salmonella, Serratia, Shigella and Yersinia. Figure 2 shows the frequency of the different bacterial species found. E. coli represented $32.43 \%$, followed by K. pneumoniae $(26.85 \%$ ) and E. aerogenes $(25.92 \%)$ respectively. Patients from 0 to 5 years old were most affected by the 3 bacterial species previously mentioned (Figure 3a). Considering the gender, E. coli was higher on men than on women, whilst the opposite with Klebsiella pneumoniae (Figure 3b). Almost all the bacterial species according to their distribution were more frequent in 
patient of Douala Subdivision areas when compared to the four other subdivisions. Results are summarized in Figure 3c.
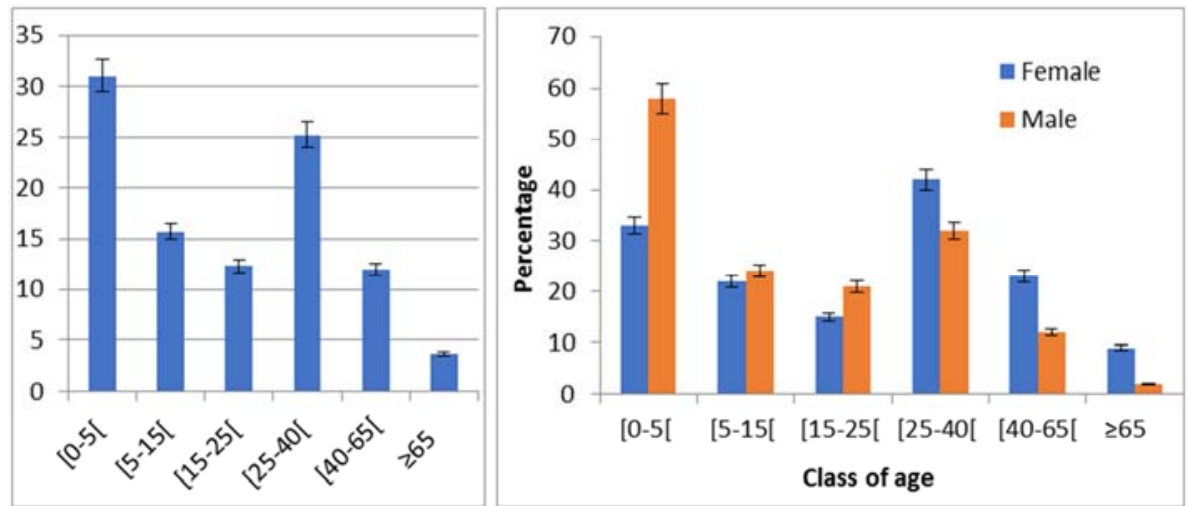

a. Distribution of patients relevant to age. b. Distribution of patients according to gender and age group.

Figure 1. Socio-demographic characteristics of the population.

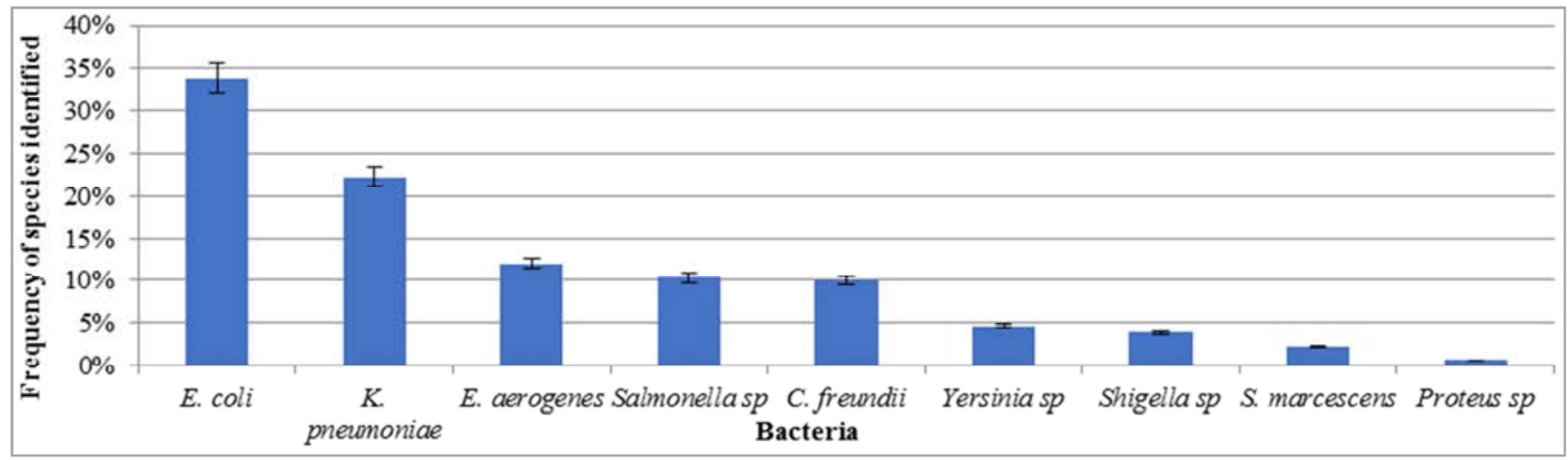

Figure 2. Frequency of bacteria identified.

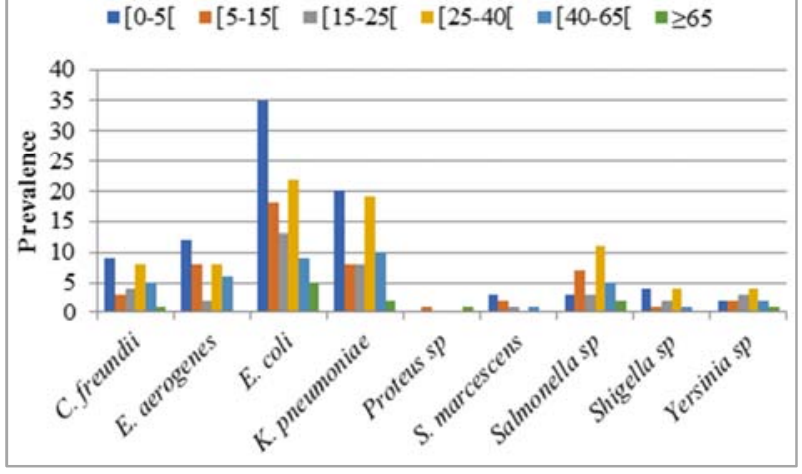

a. Frequency of appearance of bacteria species as a function of age group

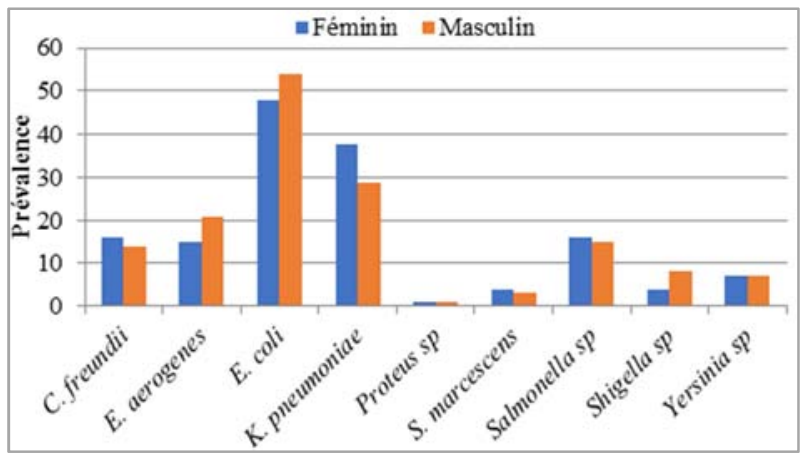

b. Frequency of appearance of bacteria species as a function of gender

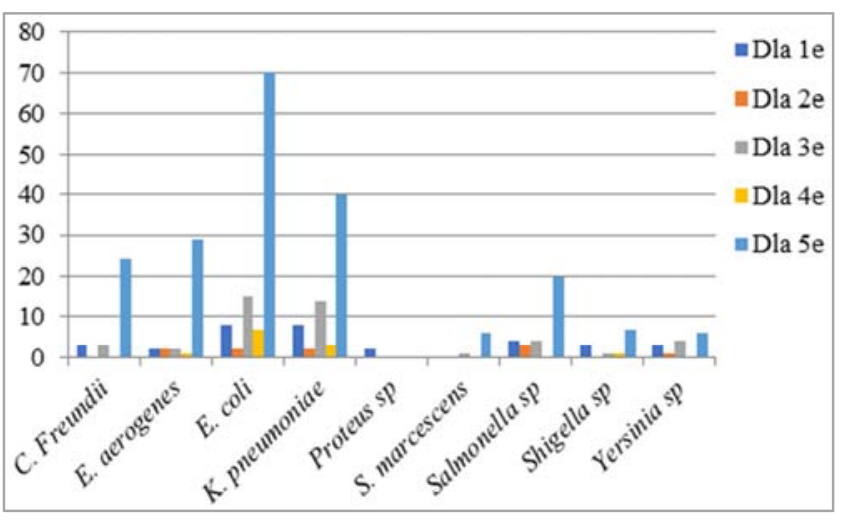

c. Frequency of appearance of bacteria species as a function of subdivision

Figure 3. Frequency of appearance of bacteria species as a function of sex, age group and subdivision.

Among patients, 129 were enrolled in the case of monoinfection $(62.62 \%)$ while $63,11,2$ and 1 patients were involved in coinfection with at least two species. Percentage of coinfections was $37.38 \%$ as reported in Figure 4a. Figure $4 \mathrm{~b}$ shows that $E$. coli was the main bacteria in coinfection with $25 \%$ followed by Klebsiella pneumoniae, and Salmonella $s p$. while Proteus sp. was the bacteria least observed, in case of coinfections with a frequency under $1 \%$. 


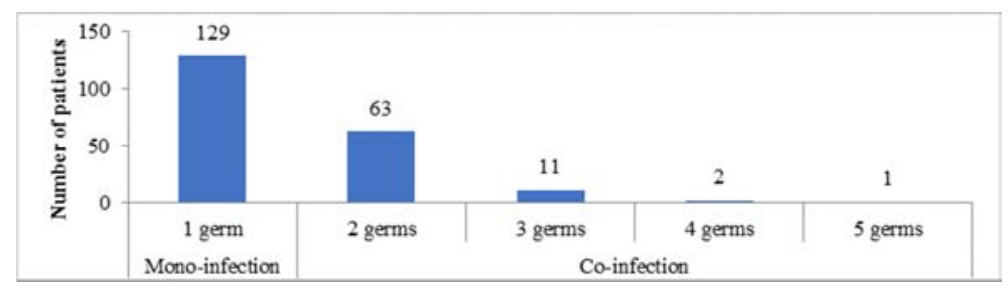

a. Frequency of patients relevant to mono and co-infection

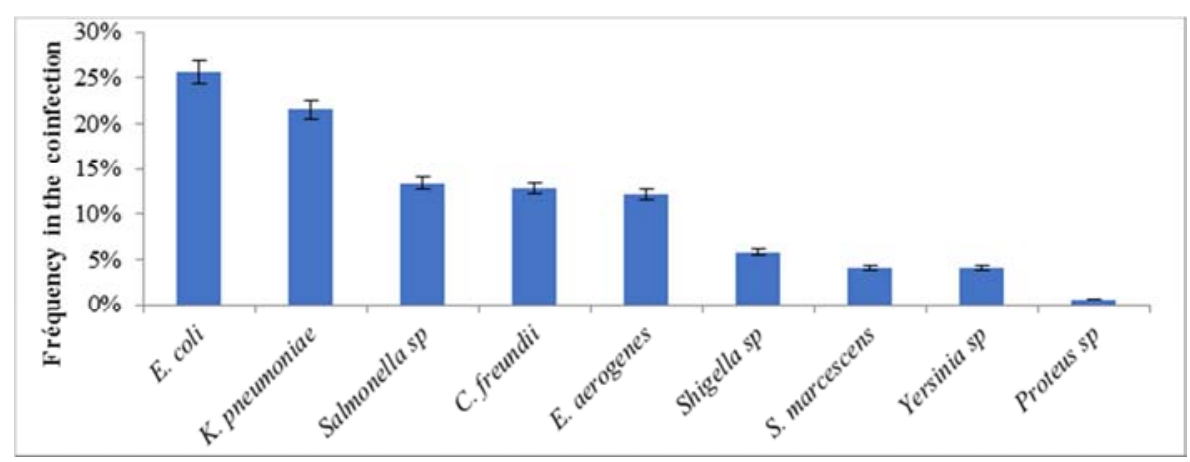

b. Frequency of different species in case of co-infection

Figure 4. Frequency of mono, co-infection and bacterial species.

\subsection{Sensibility of the Different Bacterial Species to Available Antibiotics}

The sensitivity of the different bacterial isolates to the tested antibiotics as well as their resistance pattern to each antibiotic according to sex and different classes of age are shown in Figure 5a to 13. It was found that most of the isolates were susceptible to Imipenem, Amikacin, Norfloxacin, and Ciprofloxacin. However eight out of the nine bacteria species including $E$. coli, $K$. pneumoniae, C. freundii, E. aerogenes, Yersinia sp, Salmonella sp; Shigella $s p$, Serratia marcesens showed frequent resistance to 3 different antibiotics families, including Sulfamids (Cotrimoxazol), Cyclines (Doxycycline, Tetracycline) and B-Lactamids
(Amoxycillin + Clavulanic acid, Amoxycillin, Ceftriazon and Ceftazidim) with frequency varying from one specie to another.

Isolates of $E$. coli were at $77.45 \%$ resistant with Amoxycillin, $62.75 \%$ with Amoxycillin + Clavulanic acid, 37.25\% with Ceftriaxon, $71.56 \%$ with Co-trimoxazol, $63.72 \%$ with Doxycycline and $63.72 \%$ with Tetracycline. Relevant to gender, E. coli species were at $38.3 \% ; 40 \% ; 34.4 \% ; 41.5 \% ; 40 \% ; 39.5 \%$ resistant in male with Co-trimoxazol, Doxycycline, Amoxycillin + Clavulanic, Tetracyline, Amoxycillin, Ceftriaxon respectively (Figure 5b). Particularly in children from zero to five years old, the resistance percentage of the previous seven antibiotics with E. coli species were high in male than in women (Figure $5 \mathrm{c}$ ).
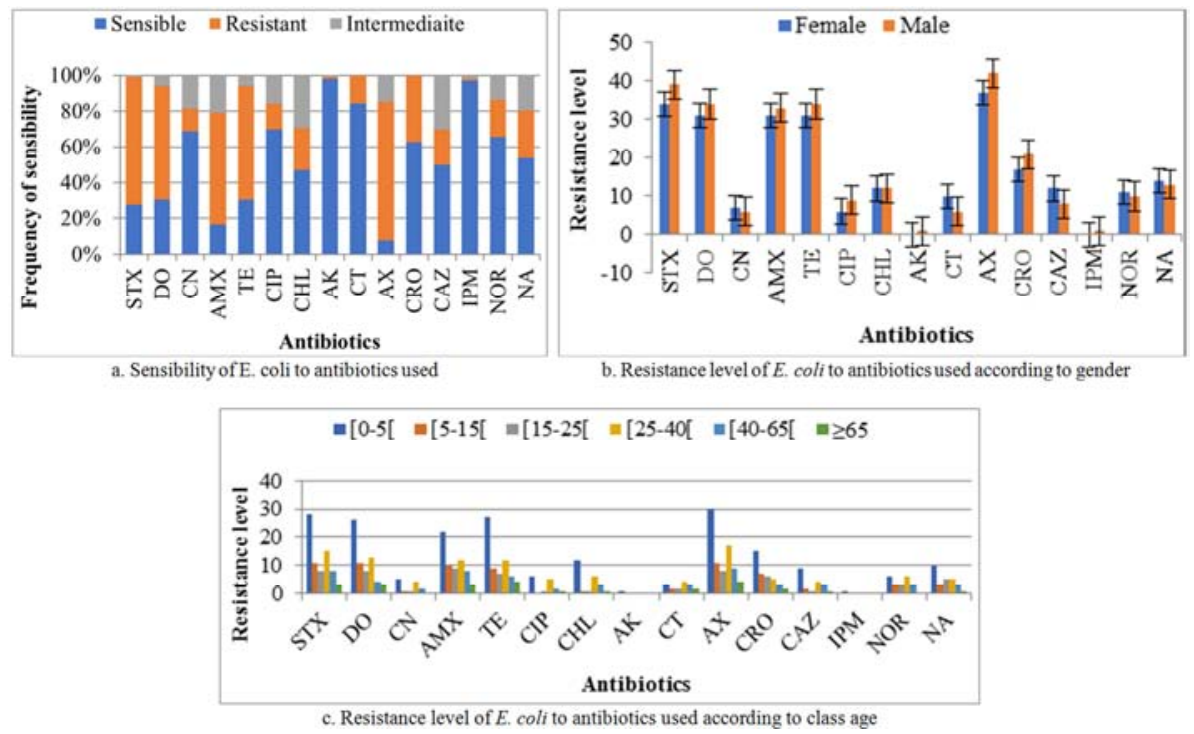

Figure 5. Sensibility and resistance profile of E. coli.

AX: Amoxicillin, AMX: Amoxicillin/clavulanic acid, CRO: Ceftriaxon, IMP: Imipenem, CAZ: Ceftazidim, CN: Gentamicin, AK: Amikacin, CT: Polymixin Colistin, STX: Cotrimoxazole, CHL: Chloramphenicol, CIP: Ciprofloxacin, NOR: Norfloxacin, NA: Nalidic Acid, TE: Tetracyclin, DO: Doxycycline 


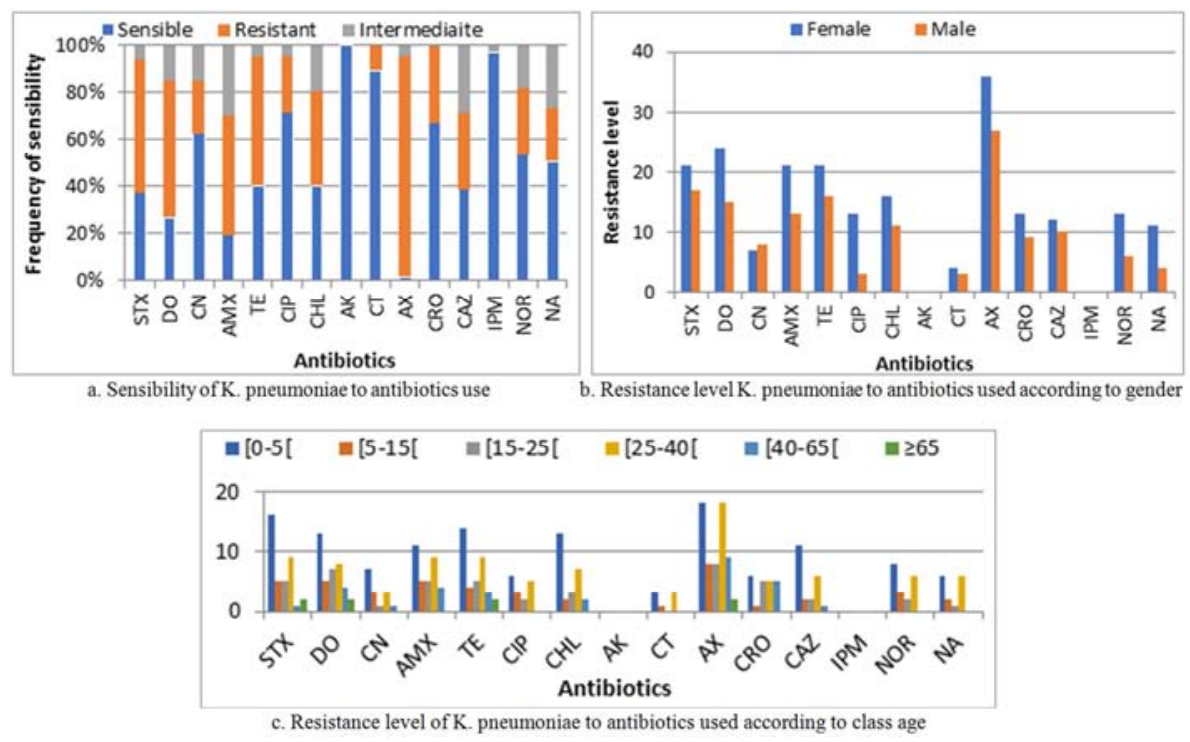

Figure 6. Sensibility and resistance profile of K. pneumoniae to antibiotics used.

AX: Amoxicillin, AMX: Amoxicillin/clavulanic acid, CRO: Ceftriaxon, IMP: Imipenem, CAZ: Ceftazidim, CN: Gentamicin, AK: Amikacin, CT: Polymixin Colistin, STX: Cotrimoxazole, CHL: Chloramphenicol, CIP: Ciprofloxacin, NOR: Norfloxacin, NA: Nalidic Acid, TE: Tetracyclin, DO: Doxycycline

Isolates of Klebsiella pneumoniae were at $94.02 \%$ resistant with Amoxycillin, 50.74\% with Amoxycillin + Clavulanic acid, $32.83 \%$ with Ceftriaxone, $40.29 \%$ with Chloramphenicol, $56.71 \%$ with Co-trimoxazol, $58.20 \%$ with Doxycycline and $55.22 \%$ with Tetracycline (Figure 6a). While relevant to gender, isolates of Klebsiella pneumoniae from women were at $55.36 \%$ resistant with Co-trimoxazol, $61.54 \%$ with Doxycycline, $61.76 \%$ with Amoxycillin +

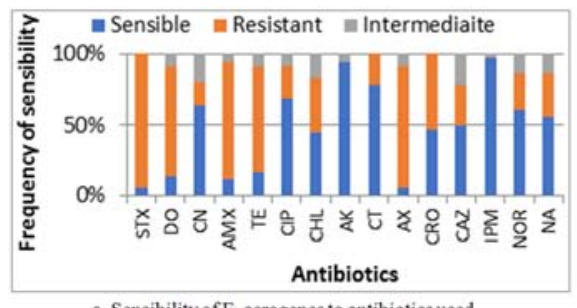

a. Sensibility of E. aerogenes to antibiotics used
Clavulanic acid, $56.75 \%$ with Tetracyline, $57.14 \%$ with Amoxycillin, 59.1\% with Ceftriaxon (Figure 6b) making resistance level higher in women than in male. Figure 6c shows that resistance level of isolates of Klebsiella pneumoniae from girls aged from zero to five years to Cotrimoxazol, Doxycycline, Amoxycillin + Clavulanic acid, Tetracyline, Amoxycillin, Ceftriaxon were high compared with boys of same age (Figure 6c).

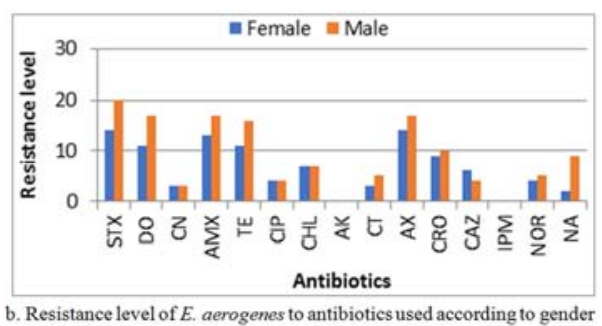

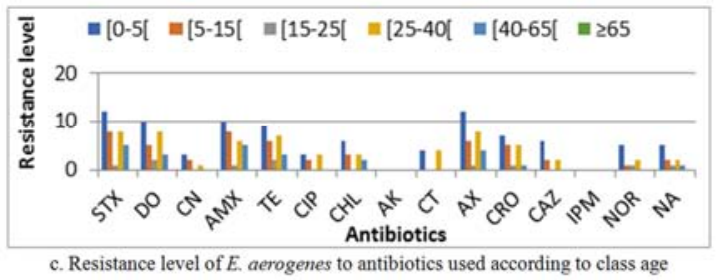

Figure 7. Sensibility and resistance profile of E. aerogenes.

AX: Amoxicillin, AMX: Amoxicillin/clavulanic acid, CRO: Ceftriaxon, IMP: Imipenem, CAZ: Ceftazidim, CN: Gentamicin, AK: Amikacin, CT: Polymixin Colistin, STX: Cotrimoxazole, CHL: Chloramphenicol, CIP: Ciprofloxacin, NOR: Norfloxacin, NA: Nalidic Acid, TE: Tetracyclin, DO: Doxycycline

Isolates of Enterobacter aerogenes were at $86.11 \%$ resistant with Amoxycillin, 83.33\% with Amoxycillin + Clavulanic acid, $52.77 \%$ with Ceftriaxon, $38.89 \%$ with Chloramphenicol, $94.44 \%$ with Co-trimoxazol, $77.77 \%$ Doxycycline and $75 \%$ with Tetracycline (Figure 7a). While relevant to gender, Isolates of Enterobacter aerogenes from male were at $58.82 \%$ resistant with Co-trimoxazol, $60.71 \%$ with Doxycyclin, and 56.67\% with Amoxycillin + Clavulanic acid 59.26\% with Tetracyline, 54.84\% with Amoxycillin, and $52.63 \%$ with Ceftriaxon (Figure $7 \mathrm{~b}$ ) making resistance level higher in men than in women. Figure $6 \mathrm{c}$ shows that resistance level of isolates of Enterobacter aerogenes from boys aged zero to five years, Co-trimoxazol, Doxycycline, Amoxycillin + Clavulanic acid, Tetracyline, Amoxycillin, 
Ceftriaxon were higher in men than in women whatever the bacteria (Figure 7c).
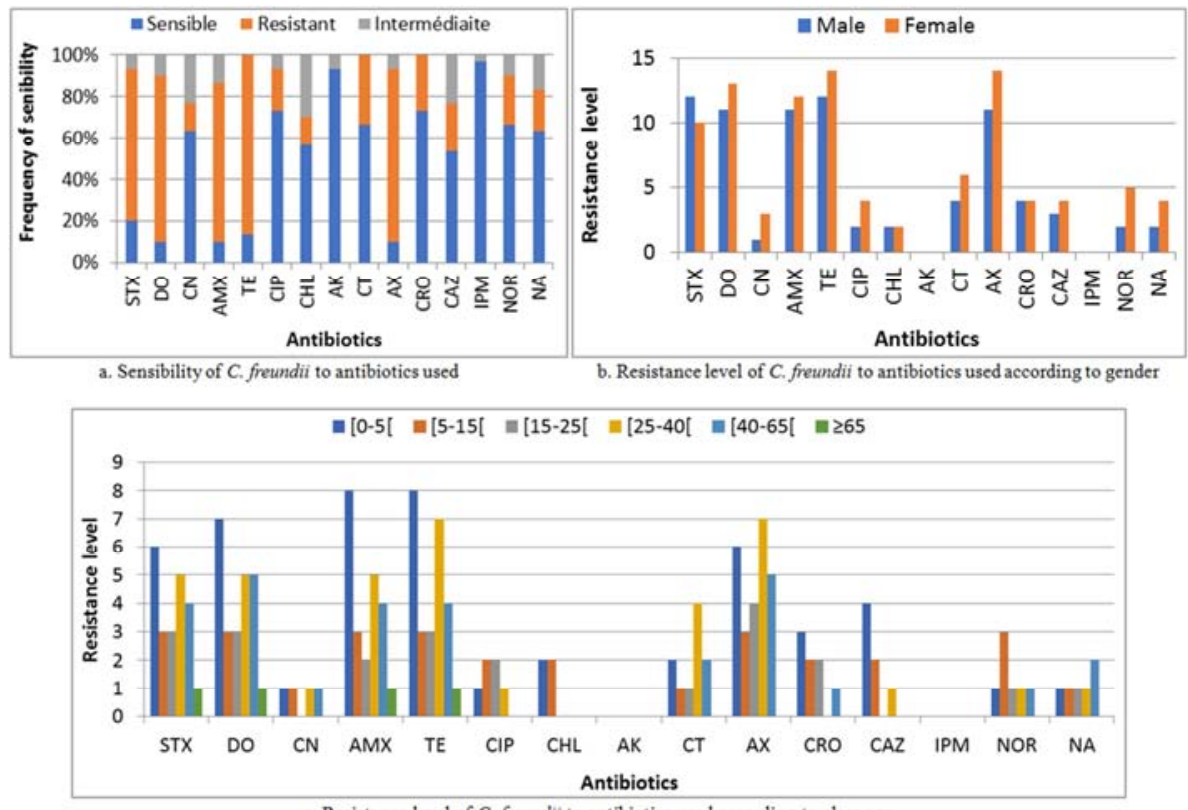

c. Resistance level of $C$. freundii to antibiotics used according to class age

Figure 8. Sensibility and resistance profile of Salmonella sp.

AX: Amoxicillin, AMX: Amoxicillin/clavulanic acid, CRO: Ceftriaxon, IMP: Imipenem, CAZ: Ceftazidim, CN: Gentamicin, AK: Amikacin, CT: Polymixin Colistin, STX: Cotrimoxazole, CHL: Chloramphenicol, CIP: Ciprofloxacin, NOR: Norfloxacin, NA: Nalidic Acid, TE: Tetracyclin, DO: Doxycycline

Resistance to Salmonella sp is summarised in Figure 8a. Isolates of Salmonella sp were at $67.74 \%$ resistant with Amoxycillin, 41.94\% with Amoxycillin + Clavulanic acid, $32.26 \%$ with Ceftriaxon, $38.71 \%$ with Co-trimoxazol, $54.84 \%$ with Doxycycline and $58.06 \%$ with Tetracycline. Resistance level in male with Cyclines (Doxycycline, Tetracyline) was at 52.94\% and $55.56 \%$ respectively while to B-Lactamids (Amoxycillin + Clavulanic acid, Amoxycillin) was at $69.3 \%$ and $57.2 \%$ in female respectively (Figure 8b). Figure $8 \mathrm{c}$ shows that this resistance was higher in patient's age from 25 to 40 years old.

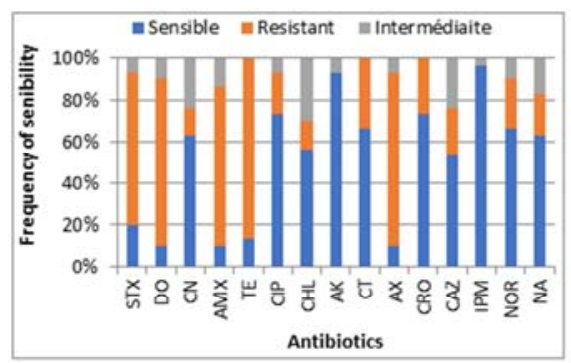

a. Sensibility of $C$. freundii to antibiotics used

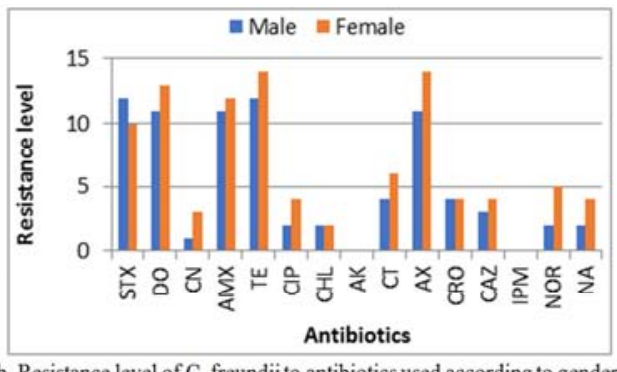

b. Resistance level of $\mathrm{C}$. freundii to antibiotics used according to gender

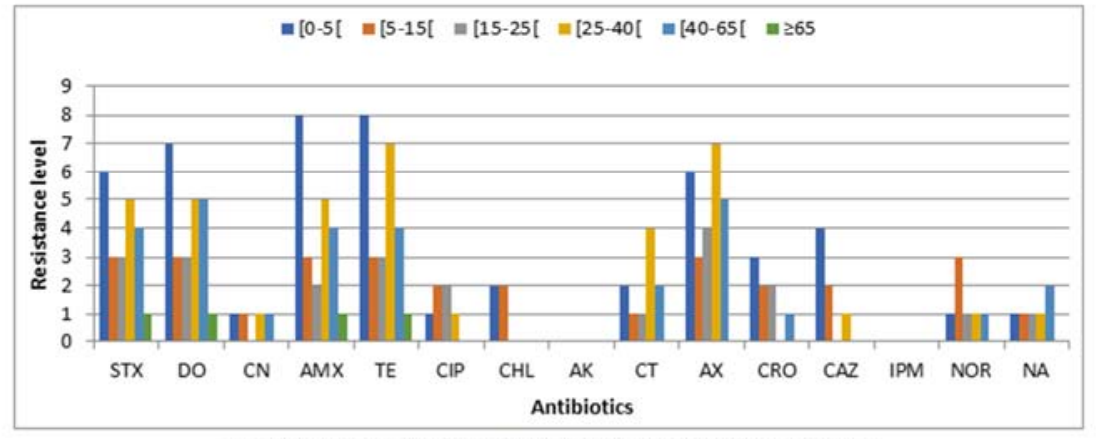

c. Resistance level of C. freundii to antibiotics used according to class age

Figure 9. Sensibility and resistance profile of C. freundii.

AX: Amoxicillin, AMX: Amoxicillin/clavulanic acid, CRO: Ceftriaxon, IMP: Imipenem, CAZ: Ceftazidim, CN: Gentamicin, AK: Amikacin, CT: Polymixin Colistin, STX: Cotrimoxazole, CHL: Chloramphenicol, CIP: Ciprofloxacin, NOR: Norfloxacin, NA: Nalidic Acid, TE: Tetracyclin, DO: Doxycycline

Figure 9a shows the resistance percentage of Citrobacter freundii

to the studied antibiotics. Refering to result obtained, Isolates of 
Citrobacter freundii were at $83.33 \%$ resistant with Amoxycillin, 76.67\% with Amoxycillin + Clavulanic acid, 73.33\% with Cotrimoxazol, $80 \%$ with Doxycycline and $86.67 \%$ with Tetracycline. Relevant to gender, isolates of Citrobacter freundii from women were resistant with Co-trimoxazol, 54.17\% with Doxycycline, 52.17\% with Amoxycillin + Clavulanic acid, 53.85\% Tetracycline

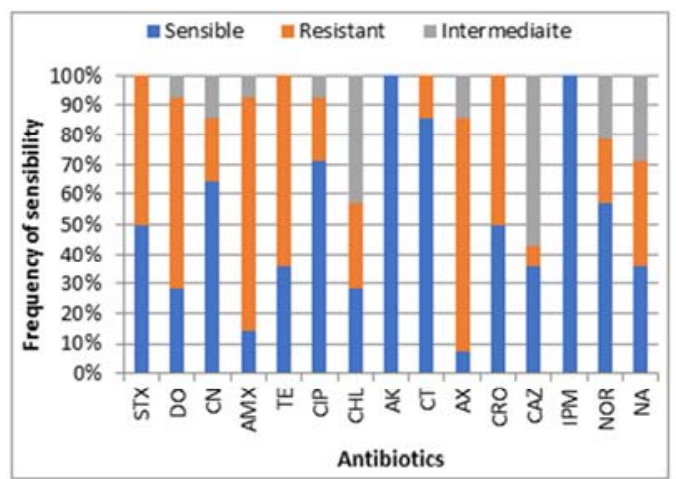

a. Sensibility of Yersinia sp to antibiotics used and 56\% with Amoxycillin. The percentage obtained in women was therefore high compared to male (Figure 9b). Relevant to group of age the same previous observations with gender were made, concerning their resistance level with Co-trimoxazol, Doxycycline, Amoxycillin + Clavulanic acid, Tetracycline, Amoxycillin among children aged from 0 to 5 years (Figure 9c).
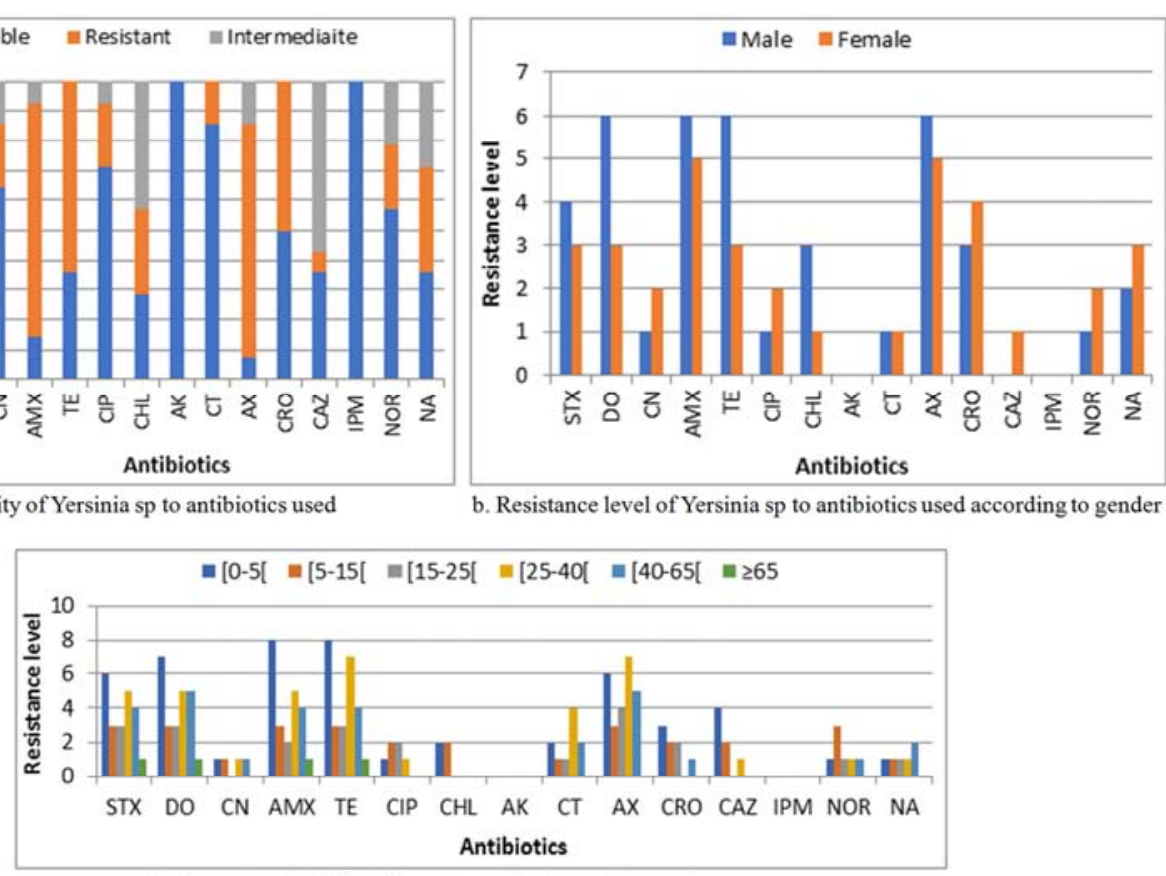

c. Resistance level of Yersinia sp to antibiotics used according to class age

Figure 10. Sensibility and resistance profile of Yersinia sp.

AX: Amoxicillin, AMX: Amoxicillin/clavulanic acid, CRO: Ceftriaxon, IMP: Imipenem, CAZ: Ceftazidim, CN: Gentamicin, AK: Amikacin, CT: Polymixin Colistin, STX: Cotrimoxazole, CHL: Chloramphenicol, CIP: Ciprofloxacin, NOR: Norfloxacin, NA: Nalidic Acid, TE: Tetracyclin, DO: Doxycycline

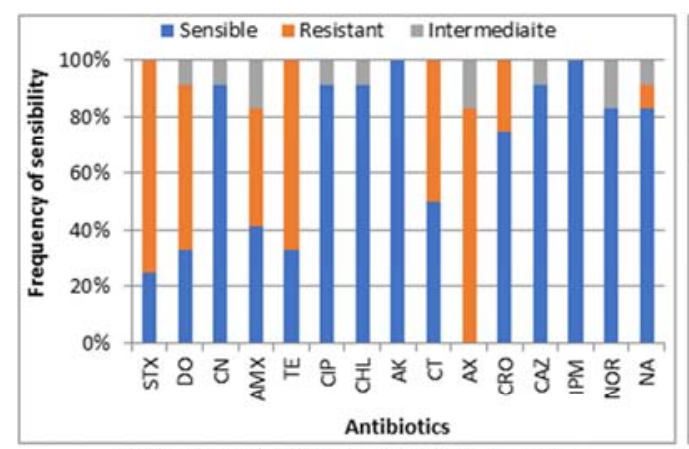

a. Sensibility of Shigella sp to antibiotics used

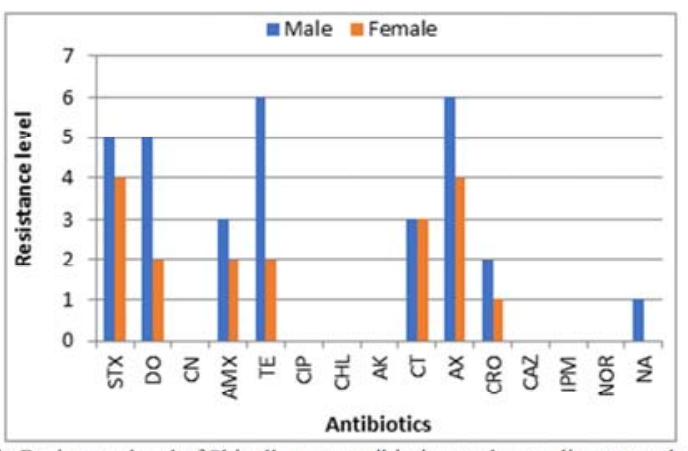

b. Resistance level of Shigella sp to antibiotics used according to gender

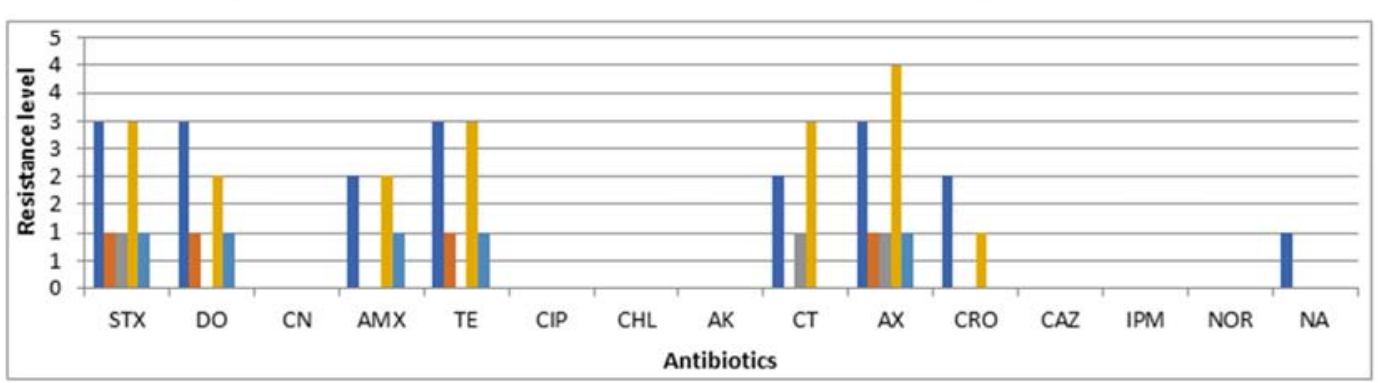

c. Resistance level of Shigella sp to antibiotics used according to class age

Figure 11. Sensibility and resistance profile of Shigella sp.

AX: Amoxicillin, AMX: Amoxicillin/clavulanic acid, CRO: Ceftriaxon, IMP: Imipenem, CAZ: Ceftazidim, CN: Gentamicin, AK: Amikacin, CT: Polymixin Colistin, STX: Cotrimoxazole, CHL: Chloramphenicol, CIP: Ciprofloxacin, NOR: Norfloxacin, NA: Nalidic Acid, TE: Tetracyclin, DO: Doxycycline 
According to Figure 10a, resistance level to Yersinia sp with Amoxycillin and Amoxycillin + Clavulanic acid was at $78.57 \%$, 50\% with Ceftriaxon and Co-trimoxazol, 64.28\% with Doxycycline and Tetracycline, and $35.71 \%$ to Nalidixic acid. Resistance level was at $57.14 \%$ with Co-trimoxazol, $66.67 \%$ with Doxycycline, Amoxycillin + Clavulanic, acid $66.67 \%$ with Amoxycillin and 54.55\% with Tetracycline (Figure 10b). Resistance level of Yersinia sp isolated from children aged from 0 to five years with Co-trimoxazol, Doxycycline, Amoxycillin + Clavulanic acid, Tetracycline,
Amoxycillin, were high in boys than in girls relevant to class of age (Figure 10c).

Figure 11a present Shigella sp resistance level. With Amoxycillin, it was $83.33 \%$, Amoxycillin + Clavulanic acid $41.66 \%$, Co-trimoxazole $75 \%$, Doxycycline $58.33 \%$, Polymixin-colistin $50 \%$ and Tetracycline $66.67 \%$. This résistance level to Co-trimoxazol, Doxycycline, Amoxycillin + Clavulanic acid, Tetracycline, Amoxycillin, Ceftriaxon was higher in male than in women (Figure 11b) and particularly in children age from 0 to five years old (Figure 12c).

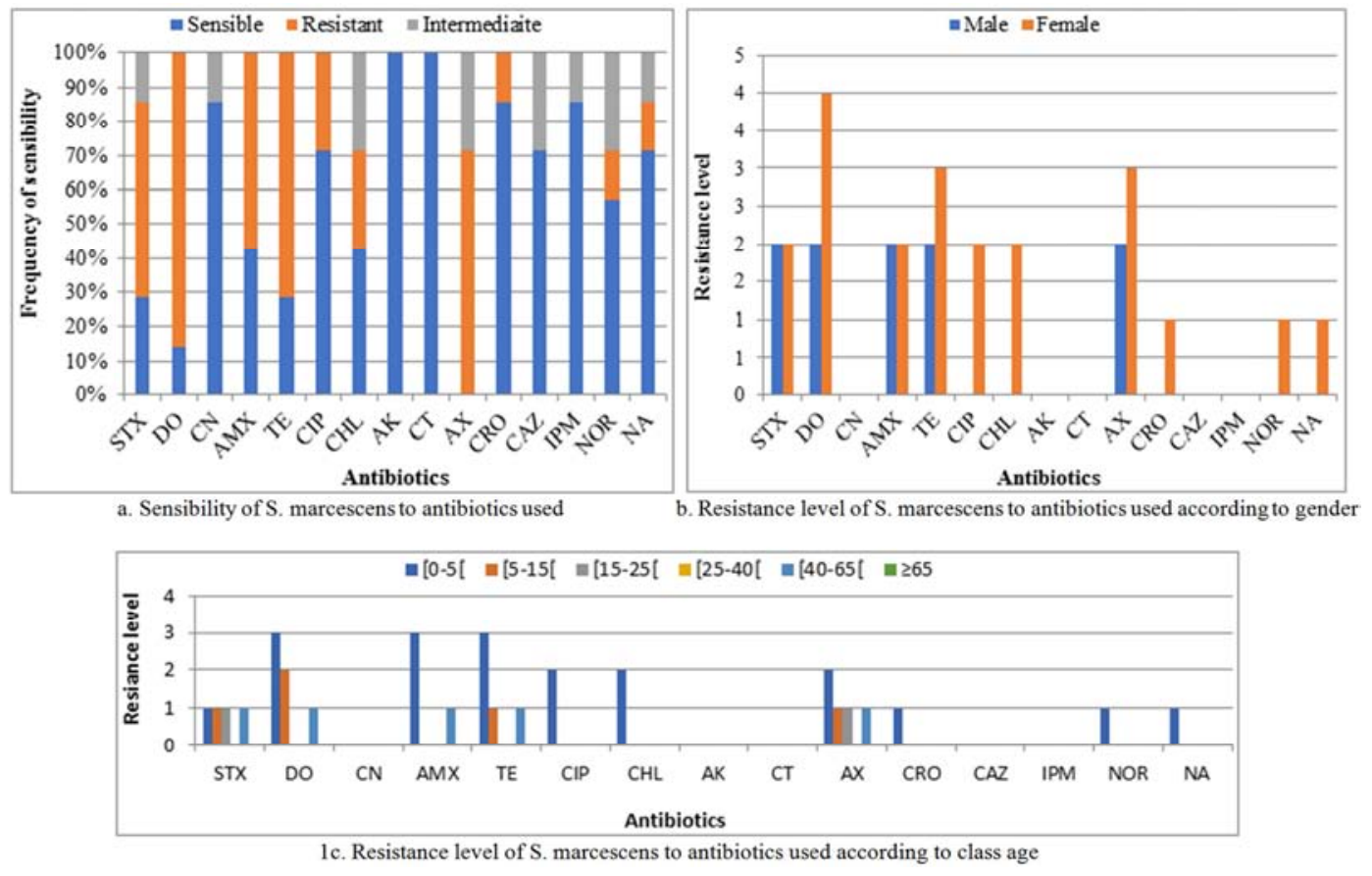

Figure 12. Sensibility and resistance profile of $S$. marcescens.

AX: Amoxicillin, AMX: Amoxicillin/clavulanic acid, CRO: Ceftriaxon, IMP: Imipenem, CAZ: Ceftazidim, CN: Gentamicin, AK: Amikacin, CT: Polymixin Colistin, STX: Cotrimoxazole, CHL: Chloramphenicol, CIP: Ciprofloxacin, NOR: Norfloxacin, NA: Nalidic Acid, TE: Tetracyclin, DO: Doxycycline

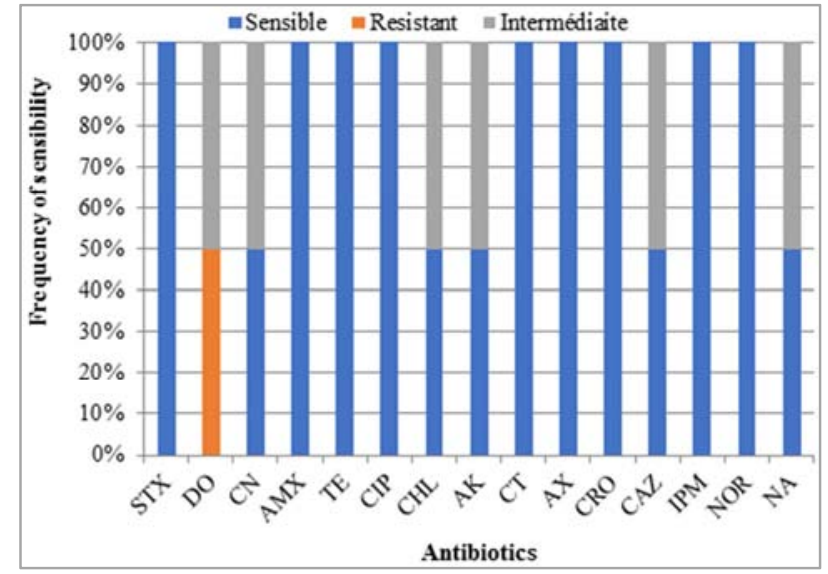

Figure 13. Sensibility and resistance profile of Proteus sp to antibiotics used.

AX: Amoxicillin, AMX: Amoxicillin/clavulanic acid, CRO: Ceftriaxon, IMP Imipenem, CAZ: Ceftazidim, CN: Gentamicin, AK: Amikacin, CT: Polymixin Colistin, STX: Cotrimoxazole, CHL: Chloramphenicol, CIP: Ciprofloxacin, NOR: Norfloxacin, NA: Nalidic Acid, TE: Tetracyclin, DO: Doxycycline
Isolates of Serratia marcesens were at $71.42 \%$ resistant with Amoxycillin, 57.14\% with Amoxycillin + Clavulanic acid, $14.28 \%$ with Ceftriaxon, $28.57 \%$ with Chloramphenicol, $57.14 \%$ with Co-trimoxazol, $85.71 \%$ with Doxycycline and $71.42 \%$ with Tetracycline (Figure 12a). According to resistance level shown in Figure 12b, Serratia marcesens resistance level with Co-trimoxazol, Doxycycline, Amoxycillin + Clavulanic acid, Tetracycline, Amoxycillin, Ceftriaxon was higher in women than in male. These Serratia marcesens isolates obtained from children of 0 to five years old were higher with Co-trimoxazol, Doxycycline, Amoxycillin + Clavulanic acid, Tetracycline, Amoxycillin, Ceftriaxon (Figure 12c).

Isolates of Proteus sp were at $71.42 \%$ resistant with Amoxycillin, 57.14\% with Amoxycillin + Clavulanic acid, $28.57 \%$ with Chloramphenicol and Ciprofloxacin, 57.14 with Co-trimoxazol, $85.71 \%$ with Doxycycline and 71.42 with Tetracycline (Figure 13).

The global resistance percentage of all the isolates shown in Figure 14 revealed that independently of bacteria, 
Imipenem and Amikacin were the most active. In the other hand a resistance percentage was observed with Ciprofloxacin and Norfloxacin known as second generation fluoroquinolons at $14.53 \%$ and $14.77 \%$ respectively.
Antimicrobial resistance profile of different clinical isolated against third generation Cephalosporins were at $12.66 \%$ with Ceftazidim and at $30.66 \%$ with Ceftriazon.

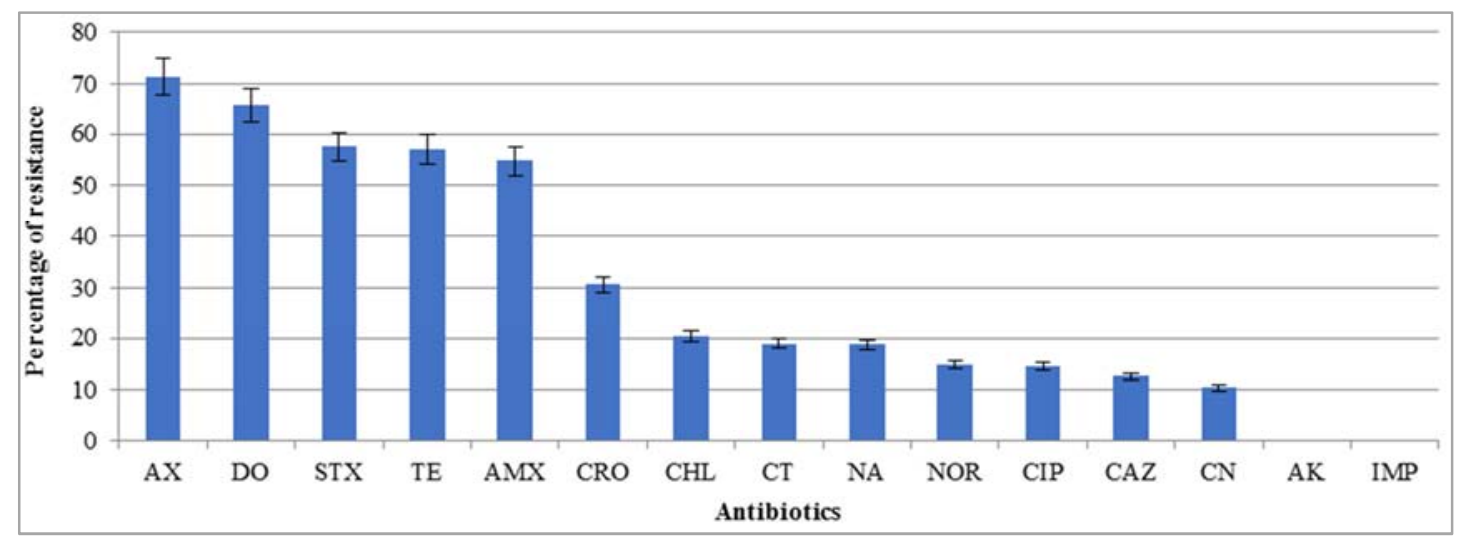

Figure 14. Global resistance percentage of species identified with antibiotics.

AX: Amoxicillin, AMX: Amoxicillin/clavulanic acid, CRO: Ceftriaxon, IMP: Imipenem, CAZ: Ceftazidim, CN: Gentamicin, AK: Amikacin, CT: Polymixin Colistin, STX: Cotrimoxazole, CHL: Chloramphenicol, CIP: Ciprofloxacin, NOR: Norfloxacin, NA: Nalidic Acid, TE: Tetracyclin, DO: Doxycycline

\section{Discussion}

In Cameroon, Infectious diseases caused by Bacteria remain one of the highest reasons for consultation or hospital visits both in children and in adults [11]. Unfortunately, there is little or no data at national level on the bacteria involved as well as their sensibility to commonly used antibiotics. While it is true that epidemiological data of some localities in the country exists [17], it remains important according to the incidence of the disease within the population to keep a constant surveillance.

A total of 206 stools samples were collected for the purpose of the present study. Douala city with its population mainly coastal and riparian is exposed to enteric diseases. This environment in the city of Douala represents a potential threat to the heath of users related to contaminated resources and the precarious conditions of general hygiene associated with the low purchasing power of the populations. 206 patients were recorded in this study, Marbou and kuete [15] obtained 105 in Mbouda while Ngalani et al., [17] obtained 148 in Dschang - West region of Cameroon. The Difference observed between the number of patients suffering from gastroenteris is in Douala and other town in Cameroon could be due to the fact that Douala is a cosmopolitan region and many water sources in Douala are unhealthy because they contain harmful physical chemicals and bacteria. However, the study revealed that the number of male patients were not different from female that visited the health facilities at the ages up to 25 years. In addition, among the studied population, children from 0 to 5 years old were most affected. This could be due to the vulnerability of children concerned and also to the consumption of contaminated water and food which contain pathogenic microorganisms. Recent studies focus on prevalence of gastro-intestinal pathogens in Sub Saharan Africa by Flechert et al. [18] who found a high incidence of pathogenic organisms, especially in children in the region when compared with the rest of the world. The same results were obtained by Mahmoud et al. [19] in Iran who focused on antimicrobial resistance patterns of Gramnegative bacteria isolated from blood team infections in an Iranian referral paediatric hospital. All these suggested that children are vulnerable worldwide. Among adults aged between 25 and 45 years, women were the most represented, this could be due to their anatomical configuration: proximity between genital and anal orifices, insufficient hygiene practices. Studies of Dougnon et al., [5] and Moutachakkir et al. [20] noted similar results on Enterobacteriaceae phenotypes responsible for community and nosochomial infections.

In the present study, 301 enterobacteriaceae from nine different genius have been isolated and identified from the stools samples. This suggests that the obtained bacteria are the principal bacteria involved in gastroenteritis in patients in this study. This can also mean that many people in Douala suffer from gastro-intestinal diseases transmitted through media as contaminated food, fingers or utensils, since it had been known that the epidemiology of gastrointestinal illness is influenced by the context in which they are transmitted. However, Ngalani et al., [17] found only five species in their study, Citrobacter, Proteus, Serratia, and Yersinia genius were not found, this suggest that other bacteria could be involved in gastrointestinal diseases and for the better diagnose of those diseases, all of them may be taken into consideration by physician. These other species have been ignored due to the scarcity of research resources, result of recent studies have shown the impact of some of them on the health of human populations, this is undoubtedly what has pushed WHO, [3] to classify some of these bacteria Proteus and Serratia among the main bacteria for which research are urgently needed. This 
suggests that an attention may be paid by clinician concerning these bacteria.

Escherichia coli $(32.42 \%)$ was the most isolated bacterial specie, followed by $K$. pneumoniae $(26.85 \%)$ and $E$. aerogenes $(25.92 \%)$. This confirms many other findings pointing $E$. coli as dominant in developing countries and transmitted mainly through faecal oral route and contaminated food or water [21]. Similar finding were revealed in Cameroon by some authors Marbou and Kuete [15]; Ngalani et al. [17] in the West region of the country. This result corroborates several studies conducted by researchers from other countries who worked on enterobacteria responsible for Urinary Tract Infections in Benin [5]. However, percentage obtained with almost all the bacterial species were lower than those obtained by Marbou and Kuete [15]; Ngalani et al. [17]. This may be due to the fact that their studies were focused on HIV patients and suggest that immunology status of patient suffering from gastro-intestinal complains may be taken into consideration. Moreover, this work shows a high presence of E. coli in children. This suggests that age could be a factor for the infection by E. coli. Dyar et al. [22] obtained similar result in rural Vietnam. The distribution of these germs according to gender and neighborhood showed that there is no association between a particular germ with the neighborhood and gender. This could be explained by the fact that in Douala, the economic capital of Cameroon, water has been identified as the main dissemination vector of germs [23], therefore, patients recruited in the present study were from a coastal environment with identical physical and chemical characteristics, characterized by salinity and porosity of coastal areas and a water table pollution outcropping. However, the conditions that govern the survival and proliferation of bacteria in neighborhoods must be more developed for a better understanding of the etiology of bacteria responsible for gastroenteritis. Salmonella sp., Escherichia coli, and Klebsiella pneumoniae were the common bacteria involved in co- infection. This could explain that gastroenteritis diseases may be due to the combined actions of at least two bacteria. Ako et al, [21] confirm that typhoid and various types of diarrhoea are among the principal enteric diseases in Douala. Gastroenteric infections (GI) could be therefore of great concern in Douala and may suggest that it is important to understand the types and prevalence of pathogens. These 3 bacteria were also the main involved in Tanzania in the study conducted by Meremo et al. [24] in febrile HIV adult patients. The involvement of these bacteria in the case of enteric infection has been demonstrated by other studies and some of them have been classified as priority bacteria against which there is an urgent need for new antibiotics [3].

The bacterial isolated showed frequency of resistance variable from one specie to another. This resistance could be acquired, in particular through the high expression of efflux pumps and more specifically those of the AcrAB-TolC type [25] or could be the consequence of the selection pressure linked to the excessive or misuse of antibiotics. The main bacteria found $E$. coli and $K$. pneumoniae showed highest resistance to Penicillin family. Similar results were found by Boutiba et al. [26] in Tunisia and Marbou and Kuete [15] in Mbouda-Cameroon and by Inan et al. [27] in India This resistance may be explained by a decrease in the activity of the beta-lactamase inhibitor (clavulanic acid), resulting from a penicillinase hyperproduction, or the inactivation of the inhibitor itself. This confirm the fact that Klebsiella pneumoniae strains are naturally resistant to Aminopenicillins due to the expression of Ambler class a chromosomal beta-lactamases [28]. This high level of resistance observed with the Penicillin class has been also demonstrated by other researchers in Douala and in Dschang $[13,17]$. The latter explained, the expression of broad spectrum $\beta$-lactamases as responsible for the resistance of isolates to several classes of $\beta$-lactams [29]. Efforts to improve patients' experiences may promote more trusting relationships and greater continuity of treatment and therefore should be a priority for physicians, educators, and health care organizations.

In the context of this study, the samples were collected in hospitals including Laquintinie Hospital which is a reference center; so, patients often come from other health facilities where probabilistic treatments based on the use of these molecules have sometimes already been initiated. Selfmedication and lack of infection management guidelines can also contribute to increase levels of resistance to these antibiotics in this context. This justifies the fact that in the present study many other bacteria relevant to class of age particularly, develop permanent resistance to Co-trimoxazole, Doxycycline, Amoxycillin + Clavulanic, Tetracyline and Amoxycillin, while some of them particularly in children from 0 to 5 years seem to develop or develop a progressive resistance to antibiotics such as Ciprofloxacin in the Quinolone class, Ceftriazon and Ceftazidim in the $\beta$-lactamins class, all them known as very active on enterobacteria. Same progressive resistance level has been observed with Gentamicin in the Aminoside class which showed a resistance of $10.43 \%$, lower than the resistance level of Ciprofloxacin (14.53\%). Since Gangoué-Piéboji et al. [11] showed that in Yaounde, enterobacteria were highly resistant to Penicillins and Cotrimoxazol: $87 \%$ of Enterobacteriaceae were resistant to amoxicillin while $73 \%$ of strains were resistant to Cotrimoxazol. This study therefore corroborated with the previous studies achieved in some other parts in the country and showed that the resistance level to these antibiotics is still growing up at an alarming rate.

Many cases of coinfections have been recorded which could explained the high resistance frequency to many antibiotics. This suggests that if nothing is not done, these antibiotics could finally have no more effect the bacteria, Therefore clinicians may pay serious attention to them in order to maintain their resistance level low. So, antibiogram exam before any antibiotic prescription could be very necessary. The multi-resistance observed with certain isolates is often associated with integrons identified in humans and animals [30]. Chang et al. [31] detected class 1 integrons harboring genes coding for resistance to Trimethoprim, 
Aminoglycosides, Chloramphenicol, and Beta-lactam antibiotics. Imipenem with $0.11 \%$ showed very low resistance level in the Carbapenem class, similarly to the resistance level of Amikacin $(0.11 \%)$ in the Aminoglycoside family. Imipenem and Amikacin therefore had good activity on enterobacterial strains. This trend has also been found by Ebongue et al. [13]; Ngalani et al. [17] in Cameroon and Dougnon et al. [5] in Benin. In contrast, Hashemi et al. [32] found higher resistance rates of around 19\% to Imipenem and $30 \%$ to Amikacinin. This could be due to the fact that they are not often used as first line antibiotics in all the context and means that physicians have a better management strategy for the use of these two antibiotics in Cameroon. They therefore could be considered as antibiotics to be taken with no rick of therapeutic failure.

\section{Conclusion}

The present study investigated bacteria species responsible for gastrointestinal infections and their resistance level to commonly used antibiotics in Douala. From the results it was found that identified pathogenic bacteria include Escherichia coli followed by Klebsiella pneumoniae, Enterobacter aerogenes, Salmonella sp, Citrobacter freundii, Yersinia enterocolitica, Shigella flexneri, Serratia marcescens and Proteus sp. The antibiogram showed high resistance to Tetracycline, Amoxycillin, Amoxycillin + clavulanic acid, Doxycycline and Cotrimoxazol. Imipenem and Amikacin were most active.

The present findings provide additional information for the control of gastrointestinal tract infections in Douala.

\section{Abbreviations}

AMR: Antimicrobial resistance; CIN: CefsulodineIrgasan-Novobiocine; EMB: Eosine Methylene Blue; MHA: Mueller Hinton Agar; WHO: World health Organization.

\section{Authors' Contributions}

RSM and RANN conceived the work, supervised the work and initiated the manuscript writing; RT, CIP, RMEE, CCND and participated in the field work. It's also confirmed that all the authors read, corrected and approved the final manuscript.

\section{Availability of Data and Materials}

Raw data were generated at Laquintinie Hospital, Deido District Hospital and Bonamoussadi District Medical Center. Thye are available from the corresponding author. The dataset supporting the conclusions of this article is included within the article.

\section{Ethics Approval and Consent to Participate}

The Institutional Ethical Committee (CEI-UDo) of the
University of Douala, Cameroon approved the study and written informed consent was obtained from the patients before collecting the samples (CEI-UDo/691/11/2016/T of $24^{\text {th }}$ November 2016).

\section{Competing Interests}

The authors declare that they have no competing interests.

\section{Acknowledgements}

The authors are highly obliged to Dr Ajonina Gordon for English revision.

\section{References}

[1] Bahramian A, Khoshnood S, Shariati A, Doustdr F, Chirani A. S, Heidary M (2019). Molecular characterization of the pilS2 gene and its association with the frequency of Pseudomonas aeruginosa plasmid pKKLC102 and PAP1-1 pathogenicity Island. Infection and Drug Resistance. 12: 221-227.

[2] Maleki D. T, Ghalavand Z, LaabeI Nikmaneh H. H, Kodori M, Haxhemi et al (2019). Molecular analysis of accessory gene regulator functionality and virulence genes in $S$. aureus derived from pediatric wound infections. Infection Genetics and Evolution. 73: 255-260.

[3] WHO: Global antimicrobial resistance surveillance system (GLASS) report: early implementation. 2017-2018 http://wwww hoint/drug resistance/surveillance/GLASS meeting/en/.

[4] Azimi T, Saied M, Fallah F, Azimi L Gholinejad Z (2019). Evaluating the antimicrobial resistance patterns among major bacterial pathogens isolated from clinical specimens taken from patients in Mofid Children's Hospital, Tehran, Iran 2013-2018. Infection and Drug Resistance. 12: 2089-2102.

[5] Dougnon V, Assogba P, Gnimatin J. P, Agbankpé J, Koudokpon H, Deguenon E (2020). Modeling the Antimicrobial Resistance of Enterobacteria Responsible for Urinary Tract Infections in Benin: Another Way to Control Antimicrobial Resistance. Asian Journal of Biochemistry, Genetics and Molecular Biology. 3: 48-61.

[6] Azimi T, Nasiri M. J, Zamani S, Hashemi A, Goudarzi H, Imani F. A. A, et al (2018). High genetic diversity among Mycobacterium tuberculosis strains in Tehran. Iranian Journal of Clinical Tuberculosis and other Mycobacterial Diseases. 11: 1-6.

[7] Aslam B, Wang W, Arshad I. M, Khursshid M, Muzammil S et al (2018). Antibiotic resistance: a rundown of a global crisis. Infection and Drug Resistance. 11: 1645-1658.

[8] Nasser A, Jazireian P, Safari H, Alizade-Sani M, Pourmand MR, Azimi T (2019). Staphylococcus aureus versus neutrophil: scrutiny of ancient combat. Microbial Pathogenesis. 131: 259-269.

[9] Tsutsui A, Suzuki S (2018). Japan nosocomial infections surveillance (JANIS) A model of sustainable national antimicrobial resistance surveillance based on hospital diagnostic microbiology laboratories. BMC Health Services Research. 18 (1): 799. 
[10] Li L, Dai J. X, Chen Z. H, Li Y, Liu M, Wen Y, et al (2018). Antimicrobial resistance and pathogen distribution in hospitalized burn patients: a multicenter study in southeast China. Medecine. 97: 34.

[11] Gangoue-Piéboji J, Koulla-Shiro S, Ngassam P,. Adiogo D, Ndumbe P (2006). Antimicrobial activity against gram negative bacilli from Yaounde Central Hospital, Cameroon. African Heath Sciences. 6 (4): 232-237.

[12] Ngueguim F (2013). Prévalence des Bactéries Gram-Négatif à Phénotypes Multi-Résistants chez les Patients souffrant d'infections du Tractus Entéro-Gastrique à l'Hôpital de District de Dschang et Rôle des Associations Des Antibiotiques. Thèse de master of science en biochimie clinique. Université de Dschang Cameroun. 104p.

[13] Ebongue C. O, Tsiazok M. D, Mefo'o J. P. N, Ngaba G. P, Beyiha G, Adiogo D (2015). Evolution of antibiotic resistance in isolated Enterobacteriaceae at the Douala General Hospital from 2005 to 2012. Panafrican Medical Jounal. 20: 227.

[14] REMIC/SFM 2015, Référentiel en microbiologie médicale Tomes 1 et $2,5^{\text {eme }}$ édition.

[15] Marbou W. J. T, Kuete V (2017). Bacterial resistance and immunological profiles in HIV- infected and non-infected patients at Mbouda AD LUCEM Hospital in Cameroon. Journal of. Infections and Public Health. 10: 269-276.

[16] Sociéte Francaise de Microbiologie 2015. (page téléchargée le 15/02/2016). Comité de l'antibiogramme: Recommandations. http://http://www.sfm.asso.fr/. Google Scholar.

[17] Ngalani J. T. O, Mbaveng A. T, Marbou W. J. T, Ngai R. Y, Kuete V (2019). Antibiotic Resistance of Enteric Bacteria in HIV-Infected Patients at the Banka Ad-Lucem Hospital, West Region of Cameroon. Canadian. Journal of. Infectious. Disesases and Medical Microbiology. 7p.

[18] Mahmoudi S, Mahzari M, Banar M, Pourakbari B, Ashtriani H. M. T, Mohammadi M, et al (2017). Antimicrobial resistance patterns of Gram-negative bacteria isolated from blood stream infections in an Iranian referral paediatric hospital: a 5.5-year study. Journal of Global Antimicrobial Resistance. 11: 17-22.

[19] Moutachakkir M, Chinbo M, Elkhoudri N, Soraa N (2015). Antibiotic resistance in uropathogenic enterobacteriaceae in pediatric settings at the CHU in Marrakech. Journal Pediatrics and Child care. 28: 16-22.

[20] Ako A. A, Nkeng G. E, Takem G. E. E (2009). Water quality and occurence of water- borne diseases in the Douala 4th District, Cameroon. Water Sciences and Technology. 59 (12): 2321-2330.

[21] Dyar O. J, Hoa N. Q, Trung N. V, Phuc H. D, Larsson M, Chuc T. K. N et al (2012). High Prevalence of antibiotic resistance in commensal Escherichia coli among children in rural Vietnam. BMC infectious Deseases. 12 (1): 92.
[22] Kweyang B. P. T, Kwetche P. R. F, Njine T, Nola M, Djogo P (2009). Quelques facteurs déterminant la distribution des bactéries du genre Vibrio dans l'environnement aquatique de Yaoundé, Cameroun. Cameroon Journal of Experimental. Biology. 5 (2): 96-103.

[23] Meremo A, Mshana S. E, Kidenya B. R, Kabangila R, Peck R, Kataraihya J. B (2012). High prevalence of non-typhoid salmonella bacteraemia among febrile HIV adult patients admitted at a tertiary hospital, North-Western Tanzania. International Archives of Medicine. 5 (1): 28.

[24] Konare M. S. Antibiotic sensitivity of enterobacteriaceae strains isolated in 2016 at the Laboratory of Medical Biology and Hospital Hygiene at CHU du Point G, 2016.

[25] Kuete V, Ngameni B, Tangmouo G, Bolla J, Alibert-Franco S, Ngadjui T, Pagès J (2010). Efflux Pumps Are Involved in the Defense of Gram-Negative Bacteria against the Natural Products Isobavachalcone and Diospyrone. Antimicrobial. Agents Chemotherapy. 54: 1749-1752.

[26] Inan A, Ozgultekin A, Akcay SS, Engin D. O, Turan G, Ceran N, Dincer E, Aksaray S, Goktas P, Erdem I (2012). Alterations in bacterial spectrum and increasing resistance rates in isolated microorganisms from device-associated infections in an intensive care unit of a Teaching Hospital in Istanbul (2004-2010). Japanese Journal of Infectious Diseases. 65 (2): $146-151$.

[27] Hæggman S, Lo"fdahl S, Paauw L. A, Verhoef J, Briss S (2004). Diversity and Evolution of the Class A Chromosomal Beta-Lactamase Gene in Klebsiella pneumoniae. Antimicrobial. Agents Chemotherapy. 48 (7): 2400-2408.

[28] Davin-Regli A, Chollet R, Boll C, Pagès J (2004). Les multirésistances et leurs mécanismes chez Enterobacter aerogenes. Antibiotherapy. 6 (3): 202-206.

[29] Gassama A, Thiaw B, Dia N. M, Fall F, Camara P, Hovette P, Perret J. L, et al (2000). Etiologies infectieuses des diarrhées au cours du SIDA de l'adulte à Dakar: Etude cas/Témoins sur 594 malades. Dakar Medical. 46: 46-50.

[30] CLSI: Performance Standards for Antimicrobial Susceptibility Testing, Clinical and Laboratory Standards Institute, Wayne, PA, USA 27th edition, 2017, https://clsi.org/media/1469/m100s27_sample.pdf.

[31] Boutiba I, Boubaker B, Ghozzi R, Jouaihia W, Mahjoubi F,. Thabet L et al (2007). Résistance bactérienne aux antibiotiques en Tunisie: données de 1999 à 2003. Revue Tunisienne d'Infectiologie. 1 (4): 5-11.

[32] Hashemi S. H, Esna-Ashari F, Tavakoli S, Mamani M (2013). The prevalence of antibiotic Resistance of Enterobacteriaceae strains isolated in community and Hospital acquired in infections in teaching hospital of Hamadan, west of Iranian. Journal of Research in Health Sciences. 13 (1): 75-80. 